Acta Cryst. (2002). A58 (Supplement), C355

EFFECTS OF MULTIPLE-WAVE INTERACTION ON X-RAY FLUORESCENCE : A SYNCHROTRON EXPERIMENT

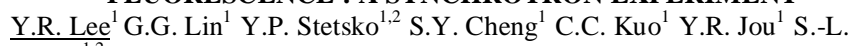
Chang $^{1,2}$

${ }^{1}$ National Tsing-Hua University Dep. of Physics 101,Section 2, Kuanf Rd. Hsinchu 300 Taiwan ${ }^{2}$ synchrotron Radiation Research Center, Hsinchu, Taiwan

Angular distribution of X-ray fluorescence under three-beam diffraction condition is investigated using synchrotron radiation for GaAs single-crystals. The fluorescence yield and multiply diffracted intensities as a function of the Bragg angle and the azimuth angle of rotation of a symmetric Bragg primary reflection are measured. It is found that fluorescence yield may increase first then decrease as the azimuth angle moves from the lower angles to higher angles across the exact three-beam diffraction position, or vice versa, depending on the polarization of the incident beam and the triplet phases involved in the diffraction process. However, the corresponding multiple diffraction intensities show almost opposite characteristic to fluorescence. The relationship between fluorescence yield and diffraction intensity is thoroughly examined. A theoretical interpretation, based on the dynamical theory, for the observed behavior is also given.

Keywords: FLUORESCENCE,MULTIPLE-WAVE,DYNAMICAL

Acta Cryst. (2002). A58 (Supplement), C355

\section{THEORY OF THE X-RAYS BACKSCATTERING BY THE CURVED CRYSTALS. MULTI-WAVE DIFFRACTION}

$\underline{\text { T. Tchen }}$

General Antonov Street, 4-1-421 MOSCOW 117279 RUSSIA

Backscattering of the X-radiation by the crystals with account for the multiwave diffraction phenomenon is theoretically investigated. The multi-wave focusing of X-Rays scattered in the backward direction is treated. Influence of the temperature on the backscattering is discussed.

\section{Keywords: BACKSCATTERING, X-RAYS, MULTI-WAVE} DIFFRACTION
Acta Cryst. (2002). A58 (Supplement), C355

PHENOMENOLOGICAL UNDERSTANDING OF THE PENDELLOESUNG

BEATS AND PROPOSAL OF ITS CONFIRMATIVE EXPERIMENT OF

DISAPPEARANCE OF THE BEATS AT LOW TEMPERATURE

T. Nakajima

Wakkanai Hokusei Gakuen College, Department of The Integrated Media, Wakabadai 2290-28 WAKKANAI 097-0013 JAPAN

Tetsuo NAKAJIMA: Hokusei Gakuen College, Faculty of the Integrated Media, Department of the Integrated Media, Wakabadai 2290- 28, Wakkanai City 097-0013, Hokkaido, JAPAN

Phenomenological Understanding of the Pendelloesung Beats and Proposal of the Confirmative Experiment of Disappearance of the Beats at Low Temperature A recoil energy loss of a target atom of the Thomson scattering in the crystal can make a reasonable offer to understand the Pendelloesung beats (PB) based upon the dynamical theory of the X-ray diffraction. A modified Bragg law, into which the recoiled absorption and emission momentums of $\Delta^{*} \mathrm{~m} * \mathrm{v}$ are introduced, is derived from the quantization of the total longitudinal momentums based upon the corpuscle model of the photon as follows:

$2 \mathrm{~d}^{*} \sin \theta=\mathrm{n} * \lambda^{*}(1+\delta)$ to the $\mathrm{p}-\mathrm{th}$,

where delta is an infinitesimal quantities called as the rate of partial charge of the recoil (RPCR) defined by the ratio of the number of the incident photons (1.01 times 10 to the 9th in the Photon Factory used for GaAs) to that of the whole crystal atoms $\left(0.01 \mathrm{~m}^{2} * 200\right.$ $\mu \mathrm{m}=8.91$ times 10 to the 20 -th for GaAs) and $\mathrm{p}$ the multiplicity of scattering, which is defined by 'the thickness of the specimen ( $200 \mu \mathrm{m}$ for GaAs) times tangent of the $\theta\left(12.4^{\circ}\right.$ for 200 plane of GaAs) divided by 200 plane spacing $(0.282 \mathrm{~nm}$ for GaAs)', $\mathrm{m}$ the single atomic mass and $\mathrm{v}$ the recoil velocity. The conventional Bragg law is derived by $\Delta=0$, as a roughest approach. From eq. (1), the relative change of the wavelength (RCWL) due to the recoil energy loss is expressed by $\mathrm{RCWL}=\Delta^{*} \mathrm{p}$, (2) which causes PB. By using $\mathrm{p}=$ 1.56 times 10 to the 5 -th, the value of eq. (2) is obtained to be 1.76 times 10 to the minus 7-th for GaAs. On the other hand, the direct experimental values of RCWL in PB is derived as $\mathrm{RCWL}=\lambda /$ extinction distance, (3) based upon the Kato-Lang relation. By using $\lambda=0.1207 \mathrm{~nm}$ and extinction distance $=50 \mu \mathrm{m}$ for GaAs, the value of eq. (3) becomes to be 1.63 times 10 to the minus 7 -th. In many cases, the necessary parameters in eq. (2) could not be obtained, because the data relevant to these parameters have not been carefully recorded in almost all works of the observation of the PB. Accidentally, from the result of the complete experiment on PB of GaAS, both values from eqs. (2) and (3) show marverously very good agreement. Further, in order to confirm the origin of PB, the observation of the temperature dependence of amplitude of $\mathrm{PB}$ from room temperature to liquid helium temperature is definitely important. As a reverse effect of the Moessbauer effect, the rigidity of the crystal is monotonically enhanced with decreasing temperature and therefore, RPCR tends to zero. Consequently, all scatterings in the diffractions become recoil-free and then, PB disappear gradually with lowering temperature. Nevertheless, if PB is observed, it could be proved that PB is thoroughly independently of the recoil in scattering.

Keywords: DYNAMICAL X-RAY DIFFRACTION, PENDELLOESUNG BEATS, RECOIL X-RAY DIFFRACTION

Acta Cryst. (2002). A58 (Supplement), C355

\section{REFRACTION IN A SELF-CONSISTENT MODEL OF X-RAY} SCATTERING

D. S. de Vasconcelos W. A. Keller

Universidade Federal Da Bahia Instituto De Fisica Rua Barao De Geremoabo, S/N, Campus De Ondina Ondina SALVADOR BAHIA 40.210-340 BRAZIL

The self-consistent approach developed initially for X-ray diffraction in perfect crystals was used to redefine the index of refraction as a dynamic parameter. It appears here as a straightforward consequence of the self-consistent interaction between EM and matter, - represented by a set of parallel and equally spaced dipole planes. Remarkably, this approach can be extended, to the wavelength region where Bragg's law is no longer valid. It is used to calculate and reproduce numerical values of the index of refraction for single $\mathrm{Si}$ crystals rendering them smaller or greater than unity for X-rays and light, respectively. It has already been known that the phase lag of the forward scattered waves behind the incident wave, symbolized by the factor $-i$, is not quite accurate. This phase difference - greater than this factor should, according to Ewald, just allow for the energy to be deflected from the incident into the reflected wave. We start from the single dipole plane and then paste the solution for the EM field, obtained for one plane with those for all the others under the strict requirement of continuity of EM field with regard to the amplitudes and phases between neighboring planes. There is here no place for any change of the wave vectors inside the crystal to those outside it. We can calculate the above mentioned phase difference, satisfying energy conservation, up to the maximum computer precision. This approach is equally valid for region of light wavelengths.

Keywords: DYNAMIC THEORY, SELF-CONSISTENCY, REFRACTION 